\title{
NONLINEAR COUPLING OF OSCILLATORY MODES IN CURRENT FLOW IN SEMI-INSULATING GaAs
}

\author{
K. KARPIŃSKa AND J. LUSAKOWSKI \\ Institute of Experimental Physics, Warsaw University \\ ul. Hoża 69, 00-681 Warszawa, Poland
}

\begin{abstract}
Spontaneous current oscillations in semi-insulating (SI) GaAs sample caused by high electric field domains nucleation were perturbed by modulated illumination. Coupling between domain and photocurrent oscillations leads to quasiperiodic and frequency-locked behaviour. The observed Arnol'd tongues structure follows the Farey tree ordering and agrees with predictions of the circle map theory. We also suggest a possible mechanism responsible for the coupling of the modes.
\end{abstract}

PACS numbers: $72.20 . \mathrm{Ht}$

\section{Introduction}

Quasiperiodicity and frequency locking attained much attention during the last decade $[1,2]$. The published results were also devoted to semiconducting systems [3-5] and exploring this area seems to be very fruitful. For one thing, one can relatively easily determine sources of different oscillatory modes. For the other, the underlying processes can be well modelled by a set of differential equations of the mass-reaction type [6]. In this paper we present results of investigation of oscillatory behaviour in a current flow through a SI GaAs sample. In the experiment, spontaneous current oscillations, generated by propagation of high electric field domains, were perturbed by illumination the sample with a monochromatic light with a modulated intensity. As a result, we observe a coupling of the two modes leading to quasiperiodic or frequency locked oscillations. We show that the observed Arnol'd tongues structure agrees with the results of the circle map theory $[1,2]$. We also discuss mechanisms responsible for coupling of the modes. 


\section{Results and discussion}

The sample under investigation ( $\mathrm{Au} / \mathrm{Ge} / \mathrm{Ni}$ contacts) was a $310.35 \mathrm{~mm}^{3}$ slab of LEC grown SI GaAs with EL2 as the main compensating level. The sample was connected in series with a voltage source and a load resistor which was used to measure the current. The ac component of the current was amplified and Fourier transformed to obtain the value of the frequency of the oscillations. The sample was illuminated by $0.98 \mu \mathrm{m}$ monochromatic light beam passing through two crossed crystalline polaryzers with a rotating $\lambda / 4$ plate placed between. This allowed to obtain a sinusoidal modulation of the light intensity. The voltage applied to the sample corresponded to an average electric field of $1200 \mathrm{~V} / \mathrm{cm}$ and the temperature was $310 \mathrm{~K}$. Propagation of the domains in such experimental conditions was checked in a separate experiment using a light spot technique described in [7]. The scheme of the experiment was the following. The sample was biased by a voltage high enough to allow stable one-mode domain oscillations with the frequency $f_{0}$ equal to $35 \mathrm{~Hz}$. The sample was then illuminated by the monochromatic light beam with the modulation frequency $f_{2}$ and the amplitude of modulation $A$. The response current frequency was $f_{1}$. For a constant $A$ we measured $f_{1}$ on $f_{2}$ dependence taking 350 values of $f_{2}$. The measurements were repeated for fifteen values of $A$.

The results of the experiment in the form of the Arnol'd tongues map are shown in Fig. 1 and they can be explained by the circle map theory [1]. The winding numbers of the Arnol'd tongues follow the Farey tree construction and the width of the tongues increases with increasing the amplitude of the sinusoidal perturbation. Between the tongues quasiperiodic oscillations were observed. A transition from a mode locked state to a chaotic state is connected with crossing a critical line in $(A, \Omega)$ space and is caused by overlapping of neighbouring tongues [1]. This is manifested by subsequent disappearance of tongues. From Fig. 1 it is seen that for $A 0.8$ some tongues are seen no more which suggests that this is the critical value of the perturbation amplitude $A_{c r}$. We checked this by determining the fractal dimensions $D$ of sets which are composed of the Arnol'd tongues for different values of $A$. Following the procedure described in [5] we obtained $D$ on $A$ dependence as shown in Fig. 2. For $A$ equal to $A_{\text {cr }}$ we obtained $D$ equal to 0.87001 which is very close to the theoretically predicted value 0.868 . The results of the presented experiment suggest a nonlinear coupling between two oscillatory modes. We suspect that domain motion is related to capturing of the conduction band electrons by EL2. This comes from the fact that the electric field required for the domain creation is high enough to enable field enhanced trapping of electrons by EL2 [8-10] and that measurements of the frequency of the domain oscillations as a function of the temperature showed an activation dependence with an energy equal to $0.06 \mathrm{eV}$, the value which is to a good accuracy equal to the height of the barrier for conduction band electron capture by EL2 [11]. The other mode is created by photocurrent oscillations caused by absorption of $0.98 \mu \mathrm{m}$ light with modulated intensity. Since the light wavelength falls in the range where absorption in GaAs is mainly due to EL2 intracentre and photoionization transition [12] we assume that other light-induced processes can be neglected. Thus, illumination the 


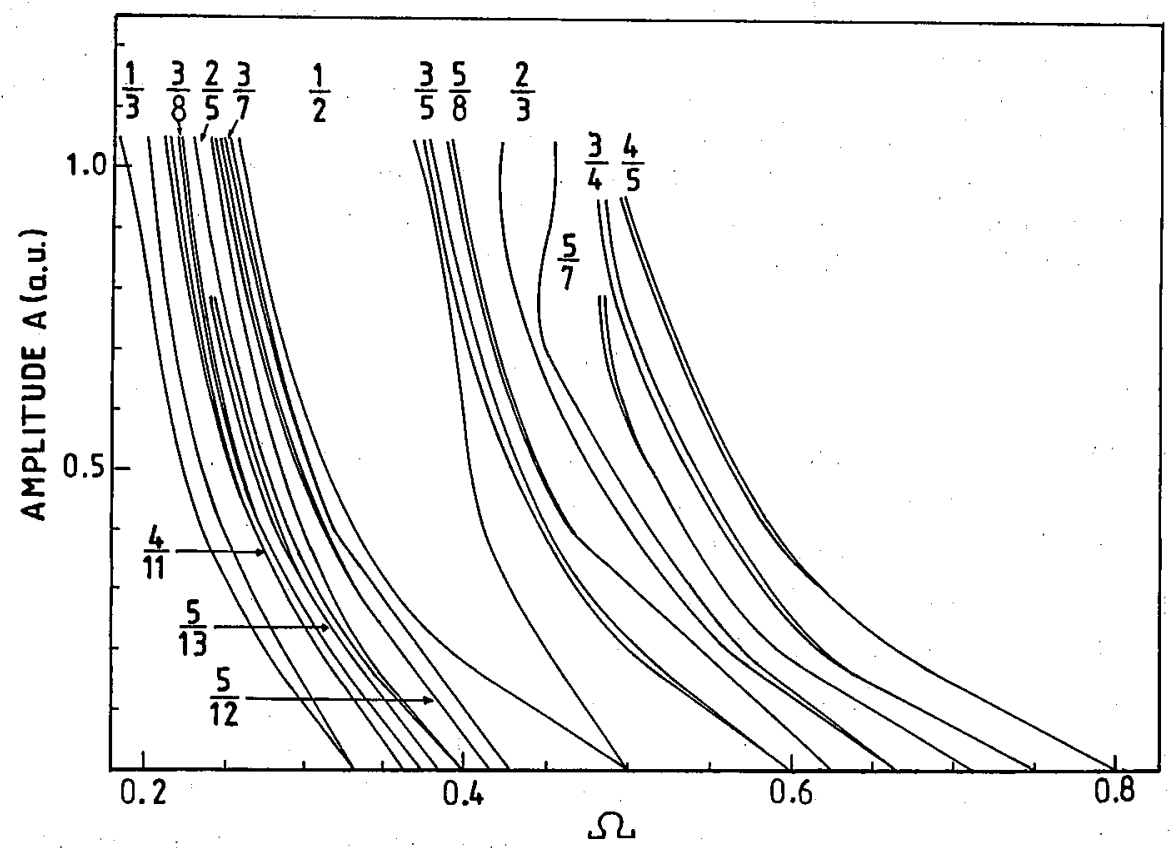

Fig. 1. The Arnol'd map of the measured current oscillations. The numbers show values of the winding numbers $(w)$ connected with each tongue. $\Omega=f_{0} / f_{2} ; w=f_{1} / f_{2}$.

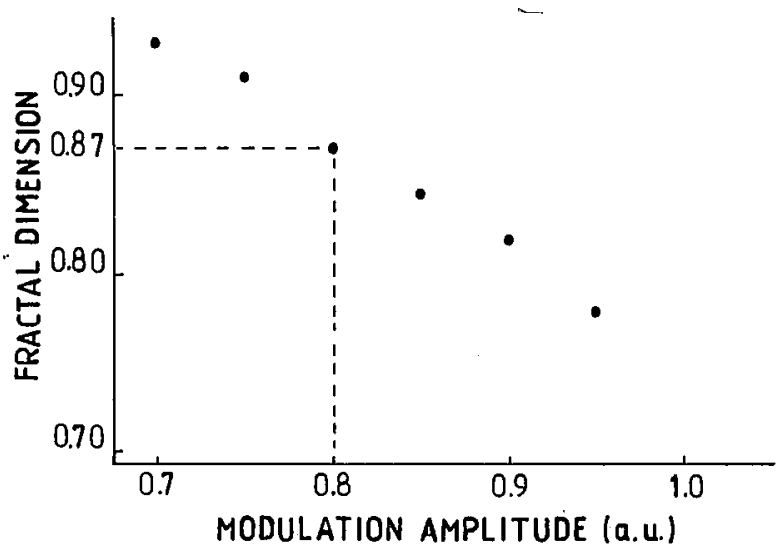

Fig. 2. The fractal dimension $D$ the section of the Arnol'd map for a constant amplitude $A$ of the sinusoidal modulation, as a function of $A$. The calculations were done for the tongues with winding numbers $2 / 3,3 / 5$ and $5 / 8$ since this is the only sequence found in the experiment which satisfies the assumptions of the adopted procedure [5]. 
sample transfers electrons from EL2 to the conduction band. In this way we are dealing with the two modes which are based on electron generation-recombination transitions between EL2 and the conduction band. A simultaneous excitation of both of them leads to a coupling which is strong enough to generate nonlinear effects responsible for quasiperiodic behaviour observed in the experiment.

The authors would like to thank Dr. A. Piotrowska and Dr. E. Kamińska, Institute of Electron Technology, Warsaw, for making the electric contacts. Discussions with Prof. M. Grynberg are gratefully acknowledged.

\section{References}

[1] M.H. Jensen, P. Bak, T. Bohr, Phys. Rev. A 30, 1960 (1984).

[2] T. Bohr, P. Bak, M.H. Jensen, Phys. Rev. A 30, 1970 (1984).

[3] H. Naber, E. Scholl, Z. Phys. B 78, 305 (1990).

[4] E.G. Gwinn, R.M. Westervelt, Phys. Rev. Lett. 57, 1060 (1986).

[5] G.A. Held, C. Jeffries, Phys. Rev. Lett. 56, 1183 (1986).

[6] F. Schlogl, Z. Phys. 253, 147 (1972).

[7] H.K. Sacks, A.G. Milnes, Int. J. Electron. 30, 49 (1971).

[8] K. Karpińska, J. Eusakowski, Acta Phys. Pol. A 79, 281 (1991).

[9] . G.C. Valley, H. Rajbenbach, H... von Bardeleben, Appl. Phys. Lett. 56, 364 (1990).

[10] M. Kamińska, J.M. Parsey, J. Lagowski, H.C. Gatos, Appl. Phys. Lett. 41, 989 (1982).

[11] P. Dreszer, M. Baj, Acta Phys. Pol. A73, 219 (1988).

[12] M. Kamińska, Rev. Phys. Appl. 23, 793 (1988). 